\title{
Robust linear discriminant analysis using S-estimators.
}

\section{Christophe CROUX and Catherine DEHON}

Key words and phrases: Breakdown point; B-Robustness; Discriminant Analysis; Influence function; Multivariate Location; S-estimators.

AMS 1991 subject classifications: Primary 62G35; secondary 62H30 .

\section{ABSTRACT}

The authors consider a robust linear discriminant function based on high breakdown location and covariance matrix estimators. They derive influence functions for the estimators of the parameters of the discriminant function and for the associated classification error. The most B-robust estimator is determined within the class of multivariate S-estimators. This estimator, which minimizes the maximal influence that an outlier can have on the classification error, is obtained. It turns out that this estimator is also the most B-robust location S-estimator. A comparison of the most B-robust estimator with the more familiar Biweight S-estimator is made.

\section{RÉSUMÉ}

Une fonction discriminante linéaire robuste basée sur des estimateurs de position et de matrice de covariance à haut point de rupture est étudiée dans ce papier. Nous obtenons les fonctions d'influence pour les estimateurs des paramètres de la fonction discriminante et pour l'erreur de classement associé. L'estimateur le plus B-robuste, qui minimise l'influence maximale qu'un point aberrant peut avoir sur l'erreur de classement, est obtenu dans la classe des S-estimateurs multivariés. Il se fait que cet estimateur est aussi le plus B-robuste S-estimateur de position. Une comparaison entre l'estimateur le plus B-robuste et le plus familier Biweight S-estimateur est réalisée.

\section{INTRODUCTION}

The major objective of the discriminant analysis problem is to classify multivariate data into different populations on the basis of a training sample for which the source populations are known. In this article, we restrict attention to the case of 2 populations and we observe one group of $p$-variate data $x_{1}, \ldots, x_{m}$ coming from population $\wp_{1}$ and following a distribution $H_{1}$ with mean $\mu_{1}$ and covariance matrix $\Sigma_{1}$. A second group of data $x_{m+1}, \ldots, x_{m+n}$ comes from population $\wp_{2}$, is distributed according to $H_{2}$ having mean $\mu_{2}$ and covariance matrix $\Sigma_{2}$. Different methods exist for constructing a rule to assign a new individual $x$ to $\wp_{1}$ or $\wp_{2}$. The most familiar is Fisher's linear discrimination rule, which is derived under the 
hypothesis that $\Sigma_{1}=\Sigma_{2}=\Sigma$ and is given by (see, e.g. Johnson \& Wichern 1998, chapter 10, p. 590)

$$
\Lambda(x)=x^{t} \lambda-\kappa
$$

where

$$
\lambda=\Sigma^{-1}\left(\mu_{1}-\mu_{2}\right) \quad \text { and } \quad \kappa=\left(\frac{\mu_{1}+\mu_{2}}{2}\right)^{t} \lambda .
$$

We assign $x \in \mathbb{R}^{p}$ to $\wp_{1}$ if

$$
\Lambda(x)>\ln \left(\frac{c_{2} \pi_{2}}{c_{1} \pi_{1}}\right)=\tau
$$

where $c_{1}$ is the cost of misclassifying a unit of group 1 and $\pi_{1}$ is the prior probability that $x$ will belong to population $\wp_{1}$. Similar definitions apply for $c_{2}$ and $\pi_{2}=$ $1-\pi_{1}$. In practice $\pi_{1}, \pi_{2}, c_{1}$ and $c_{2}$ are often not known and therefore we set $\tau=0$ throughout this paper. Note that we can rewrite (1) as

$$
\Lambda(x)=\frac{1}{2}\left\{d_{\mu_{2}, \Sigma}^{2}(x)-d_{\mu_{1}, \Sigma}^{2}(x)\right\},
$$

where $d_{\mu_{j}, \Sigma}^{2}(x)=\left(x-\mu_{j}\right)^{t} \Sigma^{-1}\left(x-\mu_{j}\right)$ is the statistical distance from $x$ to $\mu_{j}$. Observations coming from $\wp_{1}$ will in general be closer to $\mu_{1}$, leading to $\Lambda(x)>0$. If $\Lambda(x)<0$, then $x$ is closer to $\mu_{2}$ and will be classified as coming from $\wp_{2}$.

The constants $\lambda$ and $\kappa$ in (2) can easily be estimated from the data by plugging in estimators for the centers $\mu_{1}, \mu_{2}$ and the covariance matrix $\Sigma$. It is clear that since the classical estimates (the sample mean vectors and the pooled sample covariance matrix) are strongly influenced by outliers, the corresponding estimator for $\Lambda(x)$ will also be highly non robust. Randles, Broffitt, Ramberg \& Hogg (1978) did decrease the influence of outliers by using M-estimators for the means and the covariance of each population. But the weakness of an M-estimator is that its breakdown point, which is the maximal fraction of outliers that the estimator can withstand, is very low in high dimensions (Maronna 1976). Therefore, Chork \& Rousseeuw (1992) used high breakdown estimates based on the Minimum Volume Ellipsoïd estimator. In this paper, focus will be on S-estimators of multivariate location and scatter (Davies 1987; Rousseeuw \& Leroy 1987, p. 174). For a sample $x_{1}, \ldots, x_{n}$, they are defined as the couple $\left(t_{n}, C_{n}\right)$ which minimizes $\operatorname{det} C$ under the restriction

$$
\frac{1}{n} \sum_{i=1}^{n} \rho\left(\sqrt{\left(x_{i}-t\right)^{t} C^{-1}\left(x_{i}-t\right)}\right)=b
$$

over all possible pairs $(t, C)$, where $t \in \mathbb{R}^{p}$ and $C$ is a $p \times p$ symmetric positive definite matrix. The function $\rho$ needs to satisfy the following condition

(R) $\rho$ is symmetric, continuous, and non decreasing on $[0, \infty)$. Moreover, $\rho(0)=0$ and $\rho$ has a continuous derivative in all but a finite number of points.

The constant $b$ is set equal to $E_{F_{0}}[\rho(\|Z\|)]$ for $F_{0}$ the central model distribution. To get consistency at normal distributions we take $F_{0}=N\left(0, I_{p}\right)$. The most common choice for $\rho$ is the Biweight function

$$
\rho(u)=\min \left(\frac{u^{2}}{2}-\frac{u^{4}}{2 c^{2}}+\frac{u^{6}}{6 c^{4}}, \frac{c^{2}}{6}\right),
$$

where $c$ is a tuning constant to achieve the desired value of the breakdown point. We will call the corresponding estimator the Biweight S-estimator. 
In Section 2 of this paper, expressions for the influence functions of the estimators of the parameters of the discriminant function are presented. These estimators can be based on any affine equivariant location/scatter estimator $\left(t_{n}, C_{n}\right)$ possessing an influence function. We denote by

$$
\hat{\mu}_{1}=t_{m}\left(x_{1}, \ldots, x_{m}\right) \quad \text { and } \quad \hat{\mu}_{2}=t_{n}\left(x_{m+1}, \ldots, x_{m+n}\right)
$$

the estimates of $\mu_{1}$ and $\mu_{2}$. We also set

$$
\hat{\Sigma}_{1}=C_{m}\left(x_{1}, \ldots, x_{m}\right) \quad \text { and } \quad \hat{\Sigma}_{2}=C_{n}\left(x_{m+1}, \ldots, x_{m+n}\right)
$$

and pool them, viz

$$
\hat{\Sigma}_{n+m}=\frac{m \hat{\Sigma}_{1}+n \hat{\Sigma}_{2}}{m+n},
$$

to get an estimate of the common population scatter matrix $\Sigma$. Of course, other possibilities could be envisaged to increase the efficiency or robustness of the procedure. Proposals for S-estimators were made by He \& Fung (2000), and for the Minimum Covariance Determinant Estimator, by Hawkins \& McLachen (1997). However, since these improvements are specific to the selected scatter matrix estimator, we have choosen to retain the pooled estimator (3).

Since the primary goal of a discriminant analysis is to classify data, we are particularly interested in the influence of outliers on the classification error. Definitions and expressions for the corresponding influence functions are given in Section 3. A standard problem in robustness is now to search for the estimator with minimal gross-error sensitivity (defined as the supremum of the influence function). This estimator is called the most B-robust estimator. In Section 4, we obtain the most B-robust estimator within the class of S-estimators with breakdown point greater than or equal to a specified $\varepsilon^{*}$. It turns out that this estimator is also the most B-robust location S-estimator. In Section 5, a comparison of the most B-robust estimator with the more familiar Biweight S-estimator is made. Some simulation results and conclusions are presented in the last section.

\section{INFLUENCE FUNCTIONS FOR THE LINEAR DISCRIMINANT FUNCTION}

Before computing the influence function, we need to define the functionals corresponding to the estimators considered in the previous section. Let $(T, C)$ be the location/scatter functional associated to the location/scatter estimator $\left(t_{n}, C_{n}\right)$. Then $T\left(H_{m}^{1}\right)=\hat{\mu}_{1}$ and $C\left(H_{m}^{1}\right)=\hat{\Sigma}_{1}$, where $H_{m}^{1}$ is the empirical distribution function based on the observations $x_{1}, \ldots, x_{m}$. It is similar for $x_{m+1}, \ldots, x_{m+n}$ coming from $\wp_{2}$ with associated empirical distribution function $H_{n}^{2}$. We assume Fisher consistency at normal models, that is $C(N(\mu, \Sigma))=\Sigma$ and $T(N(\mu, \Sigma))=\mu$ for any multivariate normal $N(\mu, \Sigma)$. The functional associated to $\hat{\Sigma}_{m+n}$ is defined as

$$
C_{P}(G)=C\left(p_{1} G_{1}+p_{2} G_{2}\right)=p_{1} C\left(G_{1}\right)+p_{2} C\left(G_{2}\right)
$$

for any mixture distribution of the type $G=p_{1} G_{1}+p_{2} G_{2}$. At the empirical level, we take $G=p_{1} H_{m}^{1}+p_{2} H_{n}^{2}$, where $p_{1}=\frac{m}{n+m}$ and $p_{2}=1-p_{1}$.

The functional associated to the discriminant function is given by

$$
L_{x}(G)=x^{t} l(G)-k(G)
$$

with

$$
l(G)=C_{P}(G)^{-1}\left\{T\left(G_{1}\right)-T\left(G_{2}\right)\right\} \text { and } k(G)=\left\{\frac{T\left(G_{1}\right)+T\left(G_{2}\right)}{2}\right\}^{t} l(G)
$$


for any mixture distribution $G=p_{1} G_{1}+p_{2} G_{2}$.

Let $x_{0} \in \mathbb{R}^{p}$ be a possible outlier belonging to the training sample. We want to known how it will affect the value of the discriminant function. As a model distribution for the training sample, we take a mixture of two normal distributions $H_{0}=p_{1} H_{1}+p_{2} H_{2}$, where $H_{1}=N\left(\mu_{1}, \Sigma\right)$ represents the first source population and $H_{2}=N\left(\mu_{2}, \Sigma\right)$ the second. The probability that an observation of the training sample comes from $H_{1}$ is thus $p_{1}$, and $p_{2}=1-p_{1}$. Results can be extended to mixtures of elliptically symmetric distributions, but we restrict our study to the normal case. Due to Fisher consistency of the location/scatter functionals involved, we have $l\left(H_{0}\right)=\lambda$ and $k\left(H_{0}\right)=\kappa$, where $\lambda$ and $\kappa$ were defined in (2). Also, $L_{x}\left(H_{0}\right)=\Lambda(x)$ where $\Lambda(x)$ has been defined in (1).

The influence function of any statistical functional $\tilde{T}$ at the distribution $H_{0}$ is given by

$$
I F\left(x_{0}, \tilde{T}, H_{0}\right)=\lim _{\varepsilon \downarrow 0} \frac{\tilde{T}\left((1-\varepsilon) H_{0}+\varepsilon \Delta_{x_{0}}\right)-\tilde{T}\left(H_{0}\right)}{\varepsilon}
$$

where $\Delta_{x_{0}}$ is a Dirac measure putting all its mass at $x_{0}$ (see Hampel, Ronchetti, Rousseeuw \& Stahel 1986, p. 226). It can be interpreted as the infinitesimal influence that which a small amount of contamination at $x_{0}$ has on the estimator $\tilde{T}$, when the data come from the distribution $H_{0}$.

The observation $x_{0}$ is assigned to $\wp_{1}$ or $\wp_{2}$, since it enters into the training sample. This does not imply that $x_{0}$ actually arises from one of the two distributions $H_{1}$ or $H_{2}$. One needs to consider $x_{0}$ as a possible outlier, generated by an arbitrary distribution but assigned by the statistician to one of the two source distributions. As such, if $x_{0} \in \wp_{1}$, it will only influence $T\left(G_{1}\right)$ and $C\left(G_{1}\right)$, but not $T\left(G_{2}\right)$ and $C\left(G_{2}\right)$. From (5) and for $x_{0} \in \wp_{j}$, with $j \in\{1,2\}$, we have

$$
\begin{aligned}
I F\left(x_{0}, L_{x}, H_{0}\right) & =x^{t} I F\left(x_{0}, l, H_{0}\right)-\left(\frac{\mu_{1}+\mu_{2}}{2}\right)^{t} I F\left(x_{0}, l, H_{0}\right) \\
& -\lambda^{t} \frac{I F\left(x_{0}, T, H_{1}\right)+I F\left(x_{0}, T, H_{2}\right)}{2} \\
& =\left(x-\frac{\mu_{1}+\mu_{2}}{2}\right)^{t} I F\left(x_{0}, l, H_{0}\right)-\lambda^{t} \frac{I F\left(x_{0}, T, H_{j}\right)}{2} .
\end{aligned}
$$

We will first compute $\operatorname{IF}\left(x_{0}, l, H\right)$. Therefore we need the following lemma. (All proofs are collected in the Appendix.)

Lemma 1. If $x_{0} \in \wp_{j} \quad(j=1,2)$, then

$$
\operatorname{IF}\left(x_{0}, C_{P}, H_{0}\right)=\operatorname{IF}\left(x_{0}, C, H_{j}\right) .
$$

Before continuing, we need to recall Lemma 1 of Croux \& Haesbroeck (2000), which says that for every affine equivariant location/scatter functional $(T, C)$ possessing an influence function, real valued functions $\alpha$ and $\beta$ exist such that

$$
I F\left(x_{0}, C, H_{j}\right)=\alpha\left(d_{\mu_{j}, \Sigma}^{2}\left(x_{0}\right)\right)\left(x_{0}-\mu_{j}\right)\left(x_{0}-\mu_{j}\right)^{t}-\beta\left(d_{\mu_{j}, \Sigma}^{2}\left(x_{0}\right)\right) \Sigma .
$$

Furthermore, there exists another real valued function $\zeta$ such that

$$
I F\left(x_{0}, T, H_{j}\right)=\zeta\left(d_{\mu_{j}, \Sigma}^{2}\left(x_{0}\right)\right)\left(x_{0}-\mu_{j}\right),
$$

for $j=1,2$. The next proposition then follows almost immediately. 
Proposition 1. Let $x_{0} \in \wp_{j}, j=1,2$. With the notations introduced above, we have

$$
\begin{aligned}
I F\left(x_{0}, l, H_{0}\right) & =\beta\left(d_{\mu_{j}, \Sigma}^{2}\left(x_{0}\right)\right) \lambda-\alpha\left(d_{\mu_{j}, \Sigma}^{2}\left(x_{0}\right)\right) \Sigma^{-1}\left(x_{0}-\mu_{j}\right)\left(x_{0}-\mu_{j}\right)^{t} \lambda \\
& +(-1)^{j+1} \zeta\left(d_{\mu_{j}, \Sigma}^{2}\left(x_{0}\right)\right) \Sigma^{-1}\left(x_{0}-\mu_{j}\right) .
\end{aligned}
$$

The components of $\lambda$ are often used to determine which variables are discriminatary. From our Proposition 1, we see that it is sufficient to use a location/scatter functional with bounded $\beta(u), \alpha(u) u$ and $\zeta(u) \sqrt{u}$ to obtain bounded influence estimators for $\lambda$. Expressions for $\alpha, \beta$ and $\zeta$ have been derived by Lopuhäa (1989) for S-estimators, by Croux \& Haesbroeck (1999) for Minimum Covariance Determinant estimators and by Lopuhäa (2000) for reweighted versions of these estimators and can be seen to result in a bounded $I F\left(x, l, H_{0}\right)$. (For S-estimators, we require boundedness of the functions $\rho$ and $u \psi(u)$, where $\psi$ is the derivative of the $\rho$-function defining the S-estimator.) Note that for the classical sample average and covariance matrix, the $\alpha, \beta$, and $\zeta$ functions are all constant and equal to one, leading to unbounded influence estimators. It is now straightforward to compute an explicit expression for the influence function of the statistical functional corresponding to the estimator of the linear discriminant function $\Lambda(x)$.

Proposition 2. Let $x_{0} \in \wp_{j} \quad(j=1,2)$. With the notations introduced above, we have

$$
\begin{gathered}
I F\left(x_{0}, L_{x}, H_{0}\right)=\beta\left(d_{\mu_{j}, \Sigma}^{2}\left(x_{0}\right)\right) \Lambda(x)-\alpha\left(d_{\mu_{j}, \Sigma}^{2}\left(x_{0}\right)\right)\left(x-\frac{\mu_{1}+\mu_{2}}{2}\right)^{t} \Sigma^{-1}\left(x_{0}-\mu_{j}\right)\left(x_{0}-\right. \\
\left.\mu_{j}\right)^{t} \lambda-\zeta\left(d_{\mu_{j}, \Sigma}^{2}\left(x_{0}\right)\right)\left\{\frac{\lambda^{t}}{2}\left(x_{0}-\mu_{j}\right)+(-1)^{j}\left(x-\frac{\mu_{1}+\mu_{2}}{2}\right)^{t} \Sigma^{-1}\left(x_{0}-\mu_{j}\right)\right\} .
\end{gathered}
$$

Once again, we see that the form of the $\alpha, \beta$ and $\zeta$ functions will determine whether the estimator for $\Lambda(x)$ has bounded influence or not. While Propositions 1 and 2 have some interest in their own right, our primary objective is to use them for computing the influence function of the misclassification error.

\section{INFLUENCE FUNCTION FOR THE CLASSIFICATION ERROR}

In the next sections, the target quantity of interest is the classification error. When using the linear discriminant rule for classifying new observations into one of the two source populations, this quantity is of primary interest and needs to be as small as possible. The question now is to know how the classification error changes when outliers are present in the training sample. For the classical procedure some papers already addressed this problem (Lachenbruch 1966, Campbell 1978, Critchley \& Vitiello 1991). We will follow the influence function approach and obtain results applicable for robust linear discriminant analysis. We expect that a robust discriminant analysis will not increase too dramatically the classification error when outliers in the training sample are present.

Let $H=\pi_{1} N\left(\mu_{1}, \Sigma\right)+\pi_{2} N\left(\mu_{2}, \Sigma\right)$ be the distribution which generates the data to classify. Note that it is not required that $p_{1}=\pi_{1}$ and $p_{2}=\pi_{2}$, as often this does not hold in applications. So aside from the mixing proportions $p_{1}$ and $p_{2}$, this distribution is the same as the distribution $H_{0}$, corresponding to the training sample and considered in the previous section. Denote $\Pi_{12}(H)=P\left(L_{X}\left(H_{0}\right)<\right.$ $\left.0 \mid X \sim H_{1}\right)$ the misclassification probability for observations coming from $\wp_{1}$, 
and $\Pi_{21}(H)=P\left(L_{X}\left(H_{0}\right)>0 \mid X \sim H_{2}\right)$ the misclassification probability for observations coming from $\wp_{2}$. A contamination at $x_{0}$ did alter the discriminant rule and the contaminated misclassification probabilities are given by $\Pi_{12}\left(\varepsilon, x_{0}, H\right)=$ $P\left(L_{X}\left(H_{\varepsilon}\right)<0 \mid X \sim H_{1}\right)$ and $\Pi_{21}\left(\varepsilon, x_{0}, H\right)=P_{H}\left(L_{X}\left(H_{\varepsilon}\right)>0 \mid X \sim H_{2}\right)$, where $H_{\varepsilon}=(1-\varepsilon) H_{0}+\varepsilon \Delta_{x_{0}}$. Note that the distribution $H$ is not subject to contamination. Define now

$$
I F\left(x_{0}, \Pi_{12}, H\right)=\lim _{\varepsilon \downarrow 0} \frac{\Pi_{12}\left(\varepsilon, x_{0}, H\right)-\Pi_{12}(H)}{\varepsilon}=\left.\frac{\partial}{\partial \varepsilon} \Pi_{12}\left(\varepsilon, x_{0}, H\right)\right|_{\varepsilon=0} .
$$

The next proposition provides an elegant expression for the above influence function.

Proposition 3. $x_{0} \in \wp_{j} \quad(j=1,2)$ and $H=\pi_{1} N\left(\mu_{1}, \Sigma\right)+\pi_{2} N\left(\mu_{2}, \Sigma\right)$. Then

$$
I F\left(x_{0}, \Pi_{12}, H\right)=\frac{\phi\left(\frac{d_{\mu_{1}, \Sigma}\left(\mu_{2}\right)}{2}\right)}{2 d_{\mu_{1}, \Sigma}\left(\mu_{2}\right)} \zeta\left(d_{\mu_{j}, \Sigma}^{2}\left(x_{0}\right)\right) \lambda^{t}\left(x_{0}-\mu_{j}\right),
$$

where the vector $\lambda$ is defined in (2), the function $\zeta$ is defined by (8), and $\phi$ denotes the standard normal density.

In the same way, it can be shown that

$$
I F\left(x_{0}, \Pi_{21}, H\right)=\frac{-\phi\left(\frac{d_{\mu_{1}, \Sigma}\left(\mu_{2}\right)}{2}\right)}{2 d_{\mu_{1}, \Sigma}\left(\mu_{2}\right)} \zeta\left(d_{\mu_{j}, \Sigma}^{2}\left(x_{0}\right)\right) \lambda^{t}\left(x_{0}-\mu_{j}\right)=-I F\left(x_{0}, \Pi_{12}, H\right) .
$$

The expressions for the influence functions of the classification errors are explicit for a given distribution $H=\pi_{1} N\left(\mu_{1}, \Sigma\right)+\pi_{2} N\left(\mu_{2}, \Sigma\right)$. An empirical version of this influence function can be obtained by replacing the population parameters $\mu_{1}, \mu_{2}$ and $\Sigma$ by their robust estimates. Note that only the $\zeta$-function is coming into (9), and not the $\alpha$ and $\beta$-functions, implying that the location estimator only determines the form of this influence function. This may seen surprising at first sight, but even for a simple location M-estimator we have that the influence function does not depend on the auxiliary scale estimator. The reason for this is that we supposed that the underlying model distributions have equal covariance matrices, as is usual when applying linear discriminant analysis. It should be stressed that it remains important to use a robust covariance matrix estimator. The choice of the robust covariance matrix does not predetermine the misclassification error for infinitesimally small amount of outliers, but it will become important for larger amounts of contaminations. It seems natural to use the robust scatter estimators accompanying the robust location estimators. We will always use the S-estimator of scatter which is jointly defined with the S-estimator of locations (see Section 1).

One sees from (9) that if $d_{\mu_{1}, \Sigma}\left(\mu_{2}\right)$ is small, implying that $\mu_{1}$ is close to $\mu_{2}$ and that the 2 populations are harder to distinguish, then $I F\left(x_{0}, \Pi_{12}, H\right)$ will be relatively big. Proposition 3 has a further interpretation. Suppose that $x_{0} \in \wp_{1}$ and "far away" from the second population, by which we mean that $\left(\mu_{1}-\mu_{2}\right)^{t} \Sigma^{-1}\left(x_{0}-\right.$ $\left.\mu_{1}\right)$ is positive. Then the estimate of the separating hyperplane $\lambda^{t} x=\kappa$ will be attracted towards $x_{0}$, and a bigger part of $\wp_{1}$ will fall on the wrong side of the separating hyperplane, implying that $\Pi_{12}(H)$ will increase. On the other hand, more observations from $\wp_{2}$ will be correctly classified, so $\Pi_{21}(H)$ will decrease.

The criterion commonly applied to quantify the quality of the discriminant analysis procedure is total probability of misclassification, defined as

$$
\operatorname{TPM}(H)=\pi_{1} \Pi_{12}(H)+\pi_{2} \Pi_{21}(H) .
$$


We would like to say that outliers do not increase the total probability of misclassification. To assess the influence that outliers might have on the total classification error, we need to compute the influence functions once again. From Proposition 2 and (10) it follows that for $x_{0} \in \wp_{j}(j=1,2)$

$$
I F\left(x_{0}, \mathrm{TPM}, H\right)=\frac{\phi\left(\frac{d_{\mu_{1}, \Sigma}\left(\mu_{2}\right)}{2}\right)}{2 d_{\mu_{1}, \Sigma}\left(\mu_{2}\right)} \zeta\left(d_{\mu_{j}, \Sigma}^{2}\left(x_{0}\right)\right)\left(\mu_{1}-\mu_{2}\right)^{t} \Sigma^{-1}\left(x_{0}-\mu_{j}\right)\left(\pi_{1}-\pi_{2}\right) .
$$

Note that if $\pi_{1}=\pi_{2}$, outliers have a zero influence on the TPM. If they increase $\Pi_{12}(H)$, they will decrease $\Pi_{21}(H)$ by the same amount, leading to a zero effect on the TPM. Most of the time, however, $\pi_{1}$ and $\pi_{2}$ are unknown but different.

In Figure 1 we picture $I F(x, \mathrm{TPM}, H)$ for $H=\frac{2}{3} N(1,1)+\frac{1}{3} N(-1,1)$ and $x \in \wp_{j}, j=1,2$. Using the classical sample average and covariance matrix (left panel) as a starting point for the linear discriminant analysis problem, we clearly obtain an unbounded influence procedure. Observe that assigning possible outliers to the population with the higher prior probability results in an uniformly lower influence. (Negative values for $I F(x, \mathrm{TPM}, H)$ are not necessarily bad, since they will decrease the classification error.) Using the Biweight S-estimator yields redescending influence functions. Huge outliers in the training sample will not affect the estimates. An observation $x$ assigned to the larger population $\wp_{1}$ can now have a larger influence on TPM than if it had been a member of $\wp_{2}$. While Figure 1 is for a univariate setting, the same conclusions can be made in the multivariate case. This is illustrated in Figure 2, where $\operatorname{IF}(x, \mathrm{TPM}, H)$ based on the Biweight S-estimator is displayed for $H=\frac{2}{3} N\left((0,1)^{t}, I_{2}\right)+\frac{1}{3} N\left((0,-1)^{t}, I_{2}\right)$ and $x \in H_{1}$. We obtain a smooth and redescending influence function, attaining the highest values for outliers "far away" from the second population.

A one number summary for the robustness of the outlined procedure for robust linear discriminant analysis is given by the gross-error sensitivity

$$
\gamma^{*}(H)=\sup _{x_{0}} I F\left(x_{0}, \mathrm{TPM}, H\right) .
$$

Under contamination of order $\varepsilon$ in the training sample, we can say that the total classification error is bounded above by $\operatorname{TPM}(H)+\varepsilon \gamma^{*}(H)$ for small values of $\varepsilon$. It is therefore important to use estimators with small gross-error sensitivities. Note that we take the maximum of the influence function in (12), and not the sup-norm of the IF as is usually the case when defining the gross-error sensitivity. This is to stress that the major concern is the increase of the misclassification error. Mathematically, however, there will be no difference, since we can readily see from (11) that $I F\left(x_{0}, \mathrm{TPM}, H\right)=I F\left(\mu_{j}-\left(x_{0}-\mu_{j}\right)\right.$, TPM, $\left.H\right)$ for $x_{0} \in \wp_{j}(j=1,2)$, which implies that

$$
\gamma^{*}(H)=\sup _{x_{0}}\left|I F\left(x_{0}, \mathrm{TPM}, H\right)\right| .
$$

By putting $u_{0}=\Sigma^{-1 / 2}\left(x_{0}-\mu_{j}\right)$, we obtain

$$
\gamma^{*}(H)=\frac{\phi\left(\frac{d_{\mu_{1}, \Sigma}\left(\mu_{2}\right)}{2}\right)\left|\pi_{1}-\pi_{2}\right|}{2 d_{\mu_{1}, \Sigma}\left(\mu_{2}\right)} \sup _{u_{0} \in \mathbb{R}^{p}}\left[\zeta\left(\left\|u_{0}\right\|^{2}\right)\left\{\Sigma^{-1 / 2}\left(\mu_{1}-\mu_{2}\right)\right\}^{t} u_{0}\right] .
$$

Since, by the Cauchy-Schwarz inequality, $\left\{\Sigma^{-1 / 2}\left(\mu_{1}-\mu_{2}\right)\right\}^{t} u_{0} \leq\left\|u_{0}\right\| \| \Sigma^{-1 / 2}\left(\mu_{1}-\right.$ $\left.\mu_{2}\right) \|$ and equality for $u_{0} \sim \Sigma^{-1 / 2}\left(\mu_{1}-\mu_{2}\right)$, the supremum in (13) will be attained 

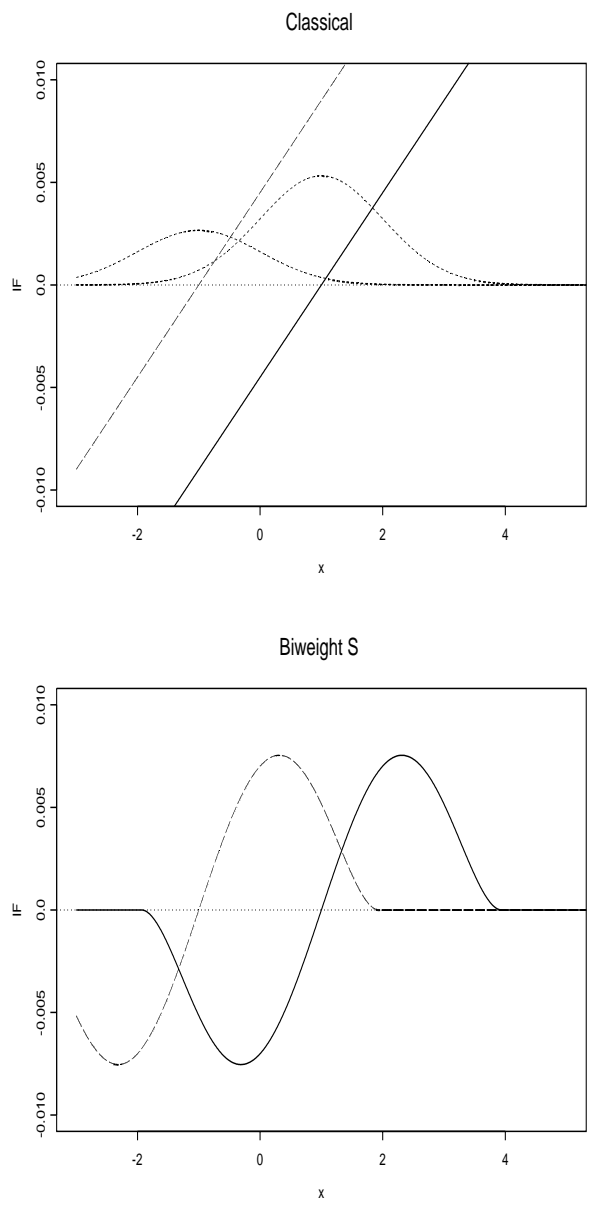

Figure 1: $\operatorname{IF}(x, T P M, H)$ for $H=\frac{2}{3} H_{1}+\frac{1}{3} H_{2}$ where $H_{1}=N(1,1)$ and $H_{2}=$ $N(-1,1)$ for linear discriminant analysis based on the classical estimators (left) and on the Biweight S-estimator (right). We distinguish between $x \in H_{1}$ (solid lines) and $x \in H_{2}$ (dashed lines). The densities of $\frac{2}{3} H_{1}$ and $\frac{1}{3} H_{3}$ are pictured in the left panel. 


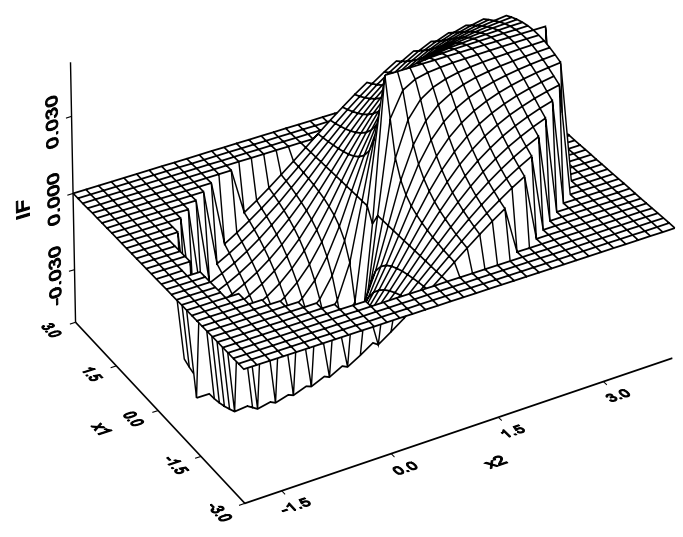

Figure 2: $I F(x, T P M, H)$ for $H=\frac{2}{3} H_{1}+\frac{1}{3} H_{2}$ where $H_{1}=N\left((0,1)^{t}, I_{2}\right)$ and $H_{2}=N\left((0,-1)^{t}, I_{2}\right)$ for linear discriminant analysis based on the Biweight $S$ estimator with $25 \%$ breakdown point for $x \in H_{1}$.

for outliers $x_{0} \in \wp_{j}$ such that $x_{0}-\mu_{j}$ is parallel to $\lambda=\Sigma^{-1}\left(\mu_{1}-\mu_{2}\right)$. The gross-error sensitivity equals thus, using $\left\|\Sigma^{-1 / 2}\left(\mu_{1}-\mu_{2}\right)\right\|=d_{\mu_{1}, \Sigma}\left(\mu_{2}\right)$,

$$
\gamma^{*}(H)=\frac{1}{2} \phi\left(\frac{d_{\mu_{1}, \Sigma}\left(\mu_{2}\right)}{2}\right)\left|\pi_{1}-\pi_{2}\right| \sup _{t \geq 0}\left\{\zeta\left(t^{2}\right) t\right\} .
$$

In the next section, we will try to find the estimator with associated $\zeta$-function which minimizes $\gamma^{*}(H)$, or equivalently minimizes $\sup _{t \geq 0}\left\{\zeta\left(t^{2}\right) t\right\}$. We will restrict our search to the class of multivariate S-estimators.

\section{MOST B-ROBUST ESTIMATORS}

The influence function for a location S-estimator has been derived by Lopuhäa (1989). It is of the form (8) with

$$
\zeta(t)=\frac{w(t)}{-2 \int w\left(\|x\|^{2}\right) g^{\prime}\left(\|x\|^{2}\right) x_{1}^{2} d x},
$$

where $g(r)=e^{-r / 2} /(2 \pi)^{p / 2}$ is such that the density of any multivariate normal $N(\mu, \Sigma)$ can be written as $g\left(d_{\mu, \Sigma}^{2}(x)\right) / \sqrt{\operatorname{det} \Sigma}$. The function $w$ is defined as $w(t)=$ $\psi(\sqrt{t}) / \sqrt{t}$ with $\psi=\rho^{\prime}$. Expression (14) can then be rewritten, using transformation to spherical coordinates, as

$$
\gamma^{*}(\psi, H)=\frac{\phi\left(\frac{d_{\mu_{1}, \Sigma}\left(\mu_{2}\right)}{2}\right)\left|\pi_{1}-\pi_{2}\right|}{4 c_{p}} \sup _{t \geq 0} \frac{\psi(t)}{-\int_{0}^{\infty} \psi(r) g^{\prime}\left(r^{2}\right) r^{p} d r}
$$

with $c_{p}=\frac{2 \pi^{p / 2}}{\Gamma(p / 2)}$. From now on, we will identify an S-estimator with its associated $\psi$-function, which is defined on the positive real line and needs to satisfy 
( $\Psi) \psi(t) \geq 0$ for every $t \geq 0$ and the set of points where $\psi$ is not continuous is finite. Moreover, $\psi$ is everywhere left continuous.

The corresponding $\rho$ function is then easily obtained as $\rho(u)=\int_{0}^{u} \psi(t) d t$. We will restrict our attention in this section to bounded $\psi$-functions. Unbounded $\psi$ functions, like $\psi(t)=t$ which corresponds to the classical estimators, yield an infinite gross-error sensitivity anyway.

Since the definition of an S-estimator is invariant to scalar multiplications of the $\psi$-function, without loss of generality we may say that $\sup _{t \geq 0} \psi(t)=1$. Minimizing $\gamma^{*}(\psi, H)$ is now equivalent with solving the problem

(P0) Maximize $-\int_{0}^{\infty} \psi(r) g^{\prime}\left(r^{2}\right) r^{p} d r$ under the restriction that $\sup _{t \geq 0} \psi(t)=1$.

The solution of problem (P0) is trivial. Since $-g^{\prime}\left(r^{2}\right) r^{p}$ and $\psi(r)$ are both nonnegative functions, the best thing to do is to take $\psi(t)=1$ for all $t$. This is also the $\psi$-function of the univariate median, which is known to be the most B-robust univariate location estimator (Rousseeuw 1982). An estimator is called most B-robust if it minimizes the gross-error sensitivity (see Rousseeuw 1982; Hampel, Ronchetti, Rousseeuw \& Stahel 1986). In higher dimensions, we will call the estimator corresponding to $\psi \equiv 1$ the $S$-median. The most B-robustness of this S-median is an optimal local robustness property (for small amounts of contamination). The global robustness (for large amounts of contamination) of the S-median is very poor, as reflected by its breakdown point.

Indeed, the breakdown point of a multivariate S-estimator is given by

$$
\varepsilon^{*}(\psi)=\min \left\{\frac{b}{\rho(\infty)}, 1-\frac{b}{\rho(\infty)}\right\}
$$

where $b=E_{F_{0}}[\rho(\|Y\|)]$ and $F_{0}=N\left(0, I_{p}\right)$. The choice $\psi \equiv 1$ yield $\rho(u)=|u|$ and therefore a zero breakdown point.

Since we want to have not only a low gross-error sensitivity but also a positive breakdown point, we add the extra restriction that $\varepsilon^{*}(\psi) \geq \varepsilon^{*}$ for a specified minimal breakdown point $0 \leq \varepsilon^{*} \leq \frac{1}{2}$. As in Hössjer (1992), we will restate this breakdown point restriction in a more tractable form. Let $\alpha=b / \rho(\infty)$, and define $G_{\alpha}(r)=G(r)-(1-\alpha)$, where $G(r)=P_{F_{0}}(\|Y\| \leq r)$ for all $r \geq 0$. So $G$ is the cumulative distribution function of a $\chi_{p}$ distribution. We have the following lemma.

Lemma 2. For any $0<\alpha<1$,

$$
E_{F_{0}}[\rho(\|Y\|)]=\alpha \rho(\infty) \text { if and only if } \int_{0}^{\infty} G_{\alpha}(r) \psi(r) d r=0 .
$$

The problem to solve becomes

(P) Maximize $-\int_{0}^{\infty} \psi(r) g^{\prime}\left(r^{2}\right) r^{p} d r$ under the restriction that $\sup _{t \geq 0} \psi(t)=1$ and $\int_{0}^{\infty} G_{\alpha}(r) \psi(r) d r=0$ for a certain $\varepsilon^{*} \leq \alpha \leq 1-\varepsilon^{*}$.

Before stating a solution to problem $(\mathbf{P})$, we need to introduce the constant $c_{\alpha}$ as the solution of

$$
\int_{0}^{c_{\alpha}} G_{\alpha}(r) d r=0,
$$




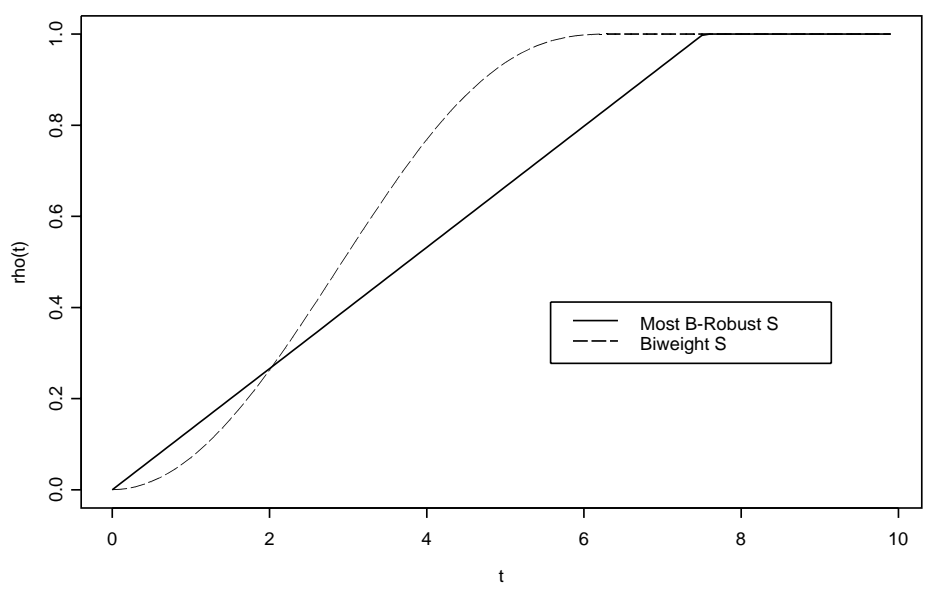

Figure 3: $\rho$-functions corresponding to the restricted most B-robust and the Biweight $S$-estimator (both with $25 \%$ breakdown point and for $p=4$ ).

for a given value of $0<\alpha<1$. Since $G_{\alpha}(t)$ is strictly increasing and continuous, $G_{\alpha}(0)<0$, and $\int_{0}^{\infty} G_{\alpha}(r) d r=\infty$, then $c_{\alpha}$ is uniquely defined. For a given $0<\varepsilon^{*} \leq 0.5$, let $c^{*}=c_{\varepsilon^{*}}$ and define

$$
\psi^{*}(t)=I\left(|t| \leq c^{*}\right),
$$

where $I(\cdot)$ stands for an indicator function. By Lemma 2 and (15), we see that $\varepsilon^{*}\left(\psi^{*}\right)$ equals the selected value for the breakdown point. The next theorem states that $\psi^{*}$ yields the most B-robust S-estimator with breakdown point larger or equal to $\varepsilon^{*}$. We will call this estimator the restricted most B-robust estimator.

Theor em 1. Let $H=p_{1} N\left(\mu_{1}, \Sigma\right)+p_{2} N\left(\mu_{2}, \Sigma\right)$ be a mixture of two normal distribution and let $0 \leq \varepsilon^{*} \leq 0.5$. For every $S$-estimator based on a $\psi$-function satisfying $(\Psi)$ and with breakdown point greater or equal to $\varepsilon^{*}$, we have

$$
\gamma^{*}(\psi, \tilde{H}) \geq \gamma^{*}\left(\psi^{*}, \tilde{H}\right),
$$

where $\psi^{*}(t)=I\left(|t| \leq c_{\varepsilon^{*}}\right)$ and $c_{\varepsilon^{*}}$ is defined by (16).

The $\rho$-function corresponding with the restricted most B-robust estimator is given by $\rho^{*}(t)=\min \left(|t|, c^{*}\right)$, which is a Huber type function. In Figure 3 we picture $\rho^{*}$ together with the Biweight $\rho$-function (both with corresponding breakdown point $25 \%$ and for $p=4$ ). The two $\rho$-functions are rescaled to have an equal supremum. One see that $\rho^{*}$ is a truncated absolute value function, while the Biweight $\rho$ resembles more a truncated quadratic loss function. Note that the most B-robust loss function attaches more weight to observations close to the origin than the Biweight $\rho$ does.

\section{THE MOST B-ROBUST LOCATION S-ESTIMATOR}

In the previous section, we constructed the most B-robust S-estimator for the linear discriminant analysis problem, minimizing the gross-error sensitivity of the total 


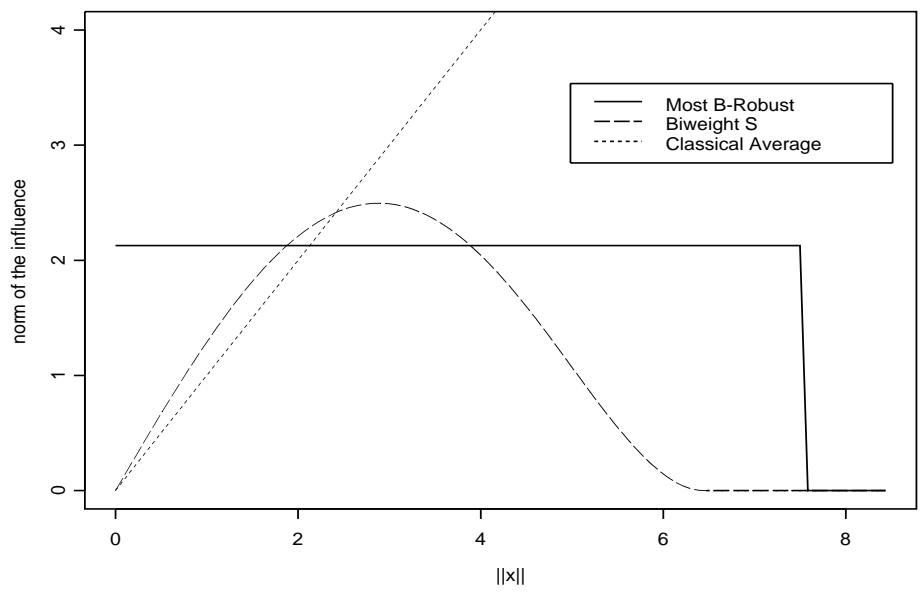

Figure 4: Norm of $\operatorname{IF}\left(x, T_{\psi}, F_{0}\right)$ as a function of $\|x\|$ for the restricted most $B$ robust, the Biweight S-estimator (both with $25 \%$ breakdown point) and the classical estimator for $p=4$.

misclassification probability. But at the same time, the gross-error sensitivity of the location part of the S-estimator is minimized. Indeed, let $T_{\psi}$ be the location S-functional associated to the chosen $\psi$ function and $F_{0}=N\left(0, I_{p}\right)$ the central model distribution. The gross-error sensitivity of $T_{\psi}$ at $F_{0}$ is defined as

$$
\gamma^{*}\left(T_{\psi}, H_{0}\right)=\sup _{x}\left\|I F\left(x, T_{\psi}, H_{0}\right)\right\| .
$$

It follows from (8) and (14) that

$$
\gamma^{*}(\psi, H)=\frac{1}{2} \phi\left(\frac{d_{\mu_{1}, \Sigma}\left(\mu_{2}\right)}{2}\right)\left|\pi_{1}-\pi_{2}\right| \gamma^{*}\left(T_{\psi}, F_{0}\right),
$$

implying that the 2 gross-error sensitivities, aside from some constants only depending on the underlying distribution, are the same. The function $\psi^{*}$ defined in (17) also yields the most B-robust location S-estimator with breakdown point greater or equal to a given $\varepsilon^{*} \in[0,0.5]$

In Figure 4, we plot the norm of $\operatorname{IF}\left(x, T_{\psi}, F_{0}\right)$ as a function of $\|x\|$ for the restricted most B-robust, Biweight S-estimator and the sample mean. (Note that the displayed functions are proportional to the $\psi$-functions.) We see that the maximal influence for the Biweight S-estimator is larger than for the restricted most B-robust estimator, but for a wide range of $x$-values the Biweight estimator yields a lower value for $\left\|I F\left(x, T_{\psi}, F_{0}\right)\right\|$. Both estimators are redescending to zero, but the $\psi^{*}$ function gives much more weight to the observations close to the center of the distribution. The Biweight influence function is closer to the influence function of the sample mean at the center of the distribution, suggesting that it will have a higher efficiency.

Let us now have a closer look at the S-median location estimator. It minimizes (under the constraint $\operatorname{det} \Sigma=1$ ) the objective function

$$
\sum_{i=1}^{n}\left\|x_{i}-t\right\|_{\Sigma}
$$


with $\|z\|_{\Sigma}^{2}=z^{t} \Sigma^{-1} z$. The above objective function resembles an $L_{1}$-criterion, using statistical distances instead of Euclidean ones. For $p=1$, the objective function becomes independent of $\Sigma$ and is minimized by the median. The second property that it shares with a median is the constant magnitude of its influence function. The main difference with the usual median is its zero breakdown point. Another disadvantage of the S-median is that, while its location part is most B-robust, its scatter part has an unbounded influence function. The S-median will merely be used as a benchmark estimator for comparing gross-error sensitivities: define for every location S-estimator the relative gross-error sensitivity (RGES) as

$$
\operatorname{RGES}\left(T_{\psi}, F_{0}\right)=\frac{\gamma^{*}\left(T_{\psi}, F_{0}\right)}{\gamma^{*}\left(\mathrm{~S}-\text { median }, F_{0}\right)} .
$$

One can check that

$$
\gamma^{*}\left(\mathrm{~S}-\text { median, } F_{0}\right)=\frac{\sqrt{2} \Gamma\left(\frac{p}{2}+1\right)}{\Gamma\left(\frac{p+1}{2}\right)},
$$

which tends to infinity with the dimension $p$. The asymptotic efficiency (for the location part, computed as in Lopuhäa 1989) of the S-median equals

$$
\operatorname{Eff}(\mathrm{S}-\text { median })=\frac{\Gamma\left(\frac{p+1}{2}\right)^{2}}{\Gamma\left(\frac{p}{2}+1\right) \Gamma\left(\frac{p}{2}\right)},
$$

and tends to $100 \%$ for $p \uparrow \infty$. This equals the relative asymptotic efficiency with respect to the sample mean, which is the Maximum Likelihood estimator at the normal location model. It is a known fact that many robust multivariate estimators tend to have optimal gaussian efficiency when the dimension increases (e.g. Croux, Dehon, Rousseeuw \& Van Aelst 2001).

In Figures 5 and 6, relative gross-error sensitivities and efficiencies for the Biweight S-estimator and the restricted most B-robust S-estimator are given as a function of their breakdown points and for several values of the dimension $p$. We only considered the Biweight S-estimators for which $0 \leq \alpha=E_{F_{0}}(\rho(\|Y\|) / \rho(\infty)=$ $\varepsilon^{*}(\psi) \leq \frac{1}{2}$. The Biweight estimators with $\alpha>\frac{1}{2}$ are more vulnerable to "implosion" and have worse efficiency and robustness behavior. The RGES of the restricted most B-robust S-estimators is very close to the optimal value 1 (for $p>1$ ). We see a conflict between local and global robustness: the higher the breakdown point, the higher $\gamma^{*}\left(\psi^{*}, F_{0}\right)$. A bit surprisingly, RGES is not monotone in $\varepsilon^{*}(\psi)$ for the Biweight S-estimator.

A trade-off between breakdown point and efficiency shows up in Figure 6. On the whole we can say that the efficiencies of both estimators are quite high for dimensions larger than 4 , even for the maximal value of the breakdown point. In the lower dimensions, it is better to take a lower value for the breakdown point to gain some extra efficiency. When $\varepsilon^{*}(\psi)$ becomes very small, the Biweight Sestimator resembles more and more the sample mean estimator. This results in an almost $100 \%$ gaussian efficiency, but also in high values for the relative gross-error sensitivity.

It is interesting to compute the limits for RGES and Eff for the dimension $p$ tending to infinity. Irrespective of the value of the breakdown point, all efficiencies (for the Biweight and restricted most B-robust S-estimators) increase to $100 \%$. The RGES of the restricted most B-robust estimators also tends to the optimal value of $100 \%$, but surprisingly this is not true for the Biweight S-estimator. For the latter estimators some calculus shows that the limit value of RGES is given 
Table 1: Simulated Bias and MSE for the restricted most B-robust location S-estimator $(M B R S)$ and the Biweight location S-estimator $(B S)$ when sampling from $N\left(0, I_{4}\right)$ and for several percentages $\varepsilon$ of intermediate and extreme outliers.

\begin{tabular}{|c|cccc|cccc|}
\hline & \multicolumn{4}{|c|}{ Bias } & \multicolumn{4}{c|}{ MSE } \\
$\varepsilon$ & \multicolumn{2}{|c}{ Intermediate } & \multicolumn{2}{c|}{ Extreme } & \multicolumn{3}{c|}{ Intermediate } & \multicolumn{2}{c|}{ Extreme } \\
& MBRS & BS & MBRS & BS & MBRS & BS & MBRS & BS \\
\hline 0 & 0.01 & 0.01 & 0.01 & 0.01 & 0.05 & 0.05 & 0.05 & 0.05 \\
0.05 & 0.10 & 0.07 & 0.12 & 0.01 & 0.06 & 0.06 & 0.10 & 0.05 \\
0.10 & 0.21 & 0.19 & 0.38 & 0.01 & 0.11 & 0.09 & 0.22 & 0.05 \\
0.20 & 0.48 & 0.51 & 1.15 & 0.04 & 0.31 & 0.31 & 1.44 & 0.11 \\
\hline
\end{tabular}

by $\left\{25 \sqrt{5} k_{\alpha}\left(1-k_{\alpha}^{2}\right)^{2} / 16\right\}^{-1}$, with $k_{\alpha}=\left\{1-(1-\alpha)^{1 / 3}\right\}^{1 / 2}$ and $\alpha$ equal to the breakdown point of the Biweight S-estimator. For small $\alpha$, the RGES for $p$ tending to infinity will be huge. For $\alpha$ larger than 0.25 , however, these limiting values are also quite close to the optimal value 1 .

A further comparison between the Biweight and the restricted most B-robust location S-estimator (abbreviated by BS and MBRS) is done by means of a modest simulation experiment. We use the $25 \%$ breakdown point version of BS and MBRS and set $p=4$. The S-estimators have been computed using the fast and accurate algorithm of Ruppert (1992). Using the latter algorithm, BS and MBRS are computed equally fast (and have the same breakdown point). For $m=1000$ samples of size $n=100$ generated from $N\left(0, I_{4}\right)$ we computed estimates $\hat{\mu}_{j}, j=1, \ldots, m$. From these, Bias and Mean Squared Error (MSE) were simulated as

$$
\operatorname{Bias}=\left\|\frac{1}{m} \sum_{j=1}^{m}\left(\hat{\mu}_{j}-\mu\right)\right\| \text { and MSE }=\frac{1}{m} \sum_{j=1}^{m}\left\|\hat{\mu}_{j}-\mu\right\|^{2},
$$

where $\mu=0$ the true location parameter. Afterwards, a certain percentage $\varepsilon$ of the data was replaced by outliers. We distinguish between extreme outliers, placed at $(\delta, 0,0,0)^{t}$ for $\delta=10$, and intermediate outliers, for which $\delta=2.88$. The intermediate outliers are located at a point where the theoretical influence function of BS is maximal. Bias and MSE for these clean and contaminated sampling schemes are tabulated in Table 1.

We see from Table 1 that the Biweight S-estimator behaves excellently for the extreme outlier configuration: a bias close to zero and almost no loss in MSE. It outperforms the MBRS estimator in the presence of extreme outliers. In the presence of intermediate outliers, the BS estimator has a higher bias and MSE, which is consistent with the form of its influence function (see Figure 4). The MBRS estimator behaves similarly to BS for intermediate outliers. Since the grosserror sensitivity of BS is only 1.17 time larger than for MBRS, the theoretical better performance of MBRS at intermediate outliers will only become apparent for big sample sizes. Finally, despite the fact that MBRS is also a redescending estimator, the bias and MSE are quite high under contamination of extreme outliers. This can be explained by the fact that the value of the rejection point $c$ of the MBRSestimator is such that $P_{F_{0}}(\|X\| \geq c) \approx 10^{-11}$, so that only very extreme outliers are completely rejected by MBRS.

As a conclusion of the comparison between the Biweight and the restricted most B-robust S-estimator, we can say that they are not so different with respect 

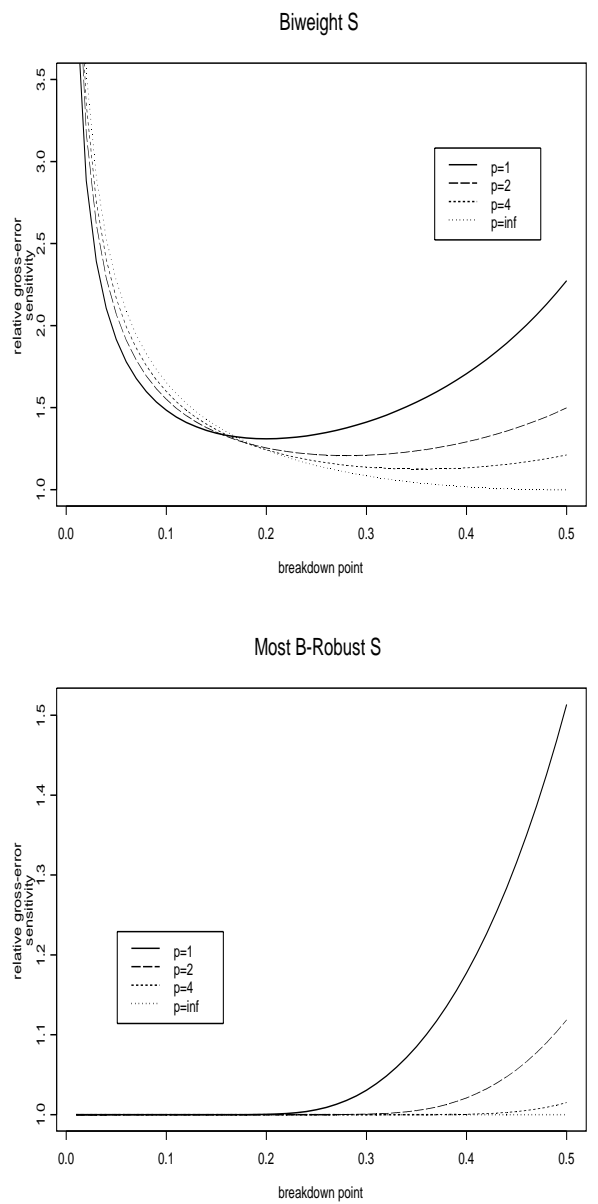

Figure 5: Relative Gross-Error Sensitivity for the Biweight S-estimator (left) and the restricted most B-robust $S$-estimator (right) as a function of their breakdown point and for several values of the dimension $p$. 

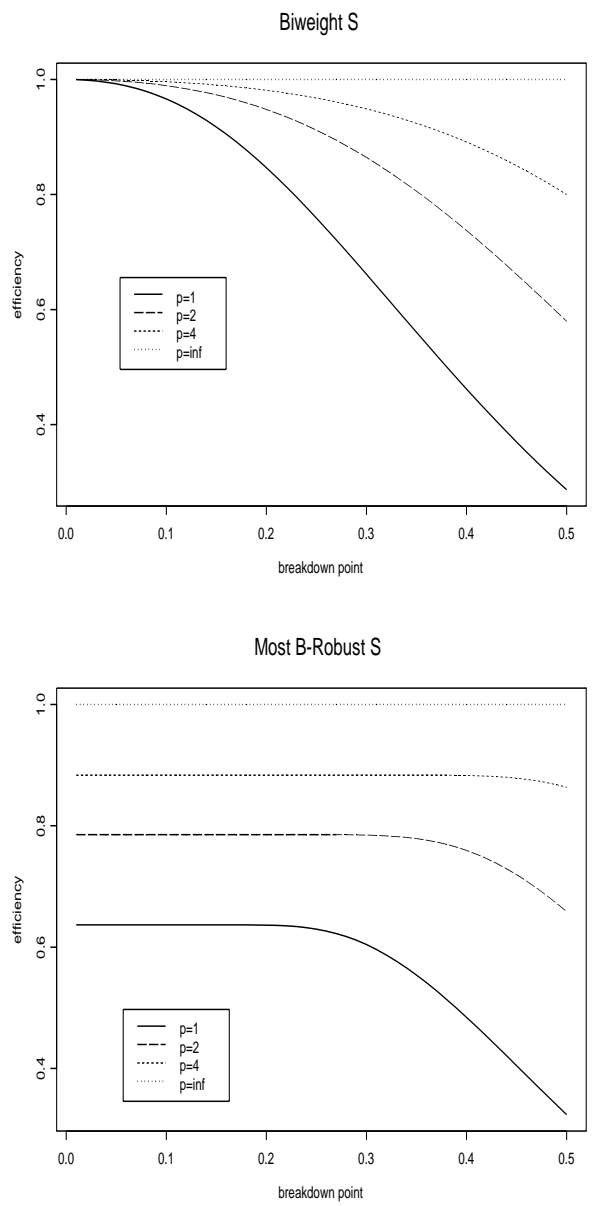

Figure 6: Efficiency of the Biweight S-estimator (left) and the restricted most Brobust S-estimator (right) as a function of their breakdown point and for several values of the dimension $p$. 
to efficiency and RGES for the common values of the breakdown point (between 0.25 and 0.50 ) if the dimension is not too small. The Biweight S-estimator is, however, much faster redescending than the Most Robust S-estimator, which is an extra safeguard against outliers, as is shown in the simulation study. Therefore we advise researchers to use the Biweight S-estimator in practical applications.

\section{CONCLUSION}

Sample averages and covariance matrices are the key ingredients to most multivariate statistical techniques. By replacing them by robust location and scatter matrices, one can robustify many multivariate procedures, as we did in this paper for discriminant analysis.

Influence functions have been computed, and the restricted most B-robust Sestimator for the linear discriminant analysis problem has been obtained. This estimator minimizes the gross-error sensitivity of the total misclassification probability. At the same time it also minimizes the gross-error-sensitivity of the location part of the S-estimator (under the same restriction).

In Section 5 the restricted most B-robust location estimator was compared with the Biweight S-estimator, and the latter one was found to be more suitable in practical applications. This observation is confirmed by a replication of the simulation study presented by He \& Fung (2000). We generated $m=1000$ training samples according to a certain mixture $p_{1} H_{1}+p_{2} H_{2}$ of 2 normal distributions in 3 dimensions. Every training sample yields a discriminant rule, which we evaluated by computing the frequency of wrongly classified observations out of a training sample of size 2000 generated from $\left(H_{1}+H_{2}\right) / 2$. This yields a sequence of $m$ estimates of the classification error, of which we computed the average and the standard deviation. In Table 2, results are shown for sampling schemes B, C, D, and E of He \& Fung (2000, p. 158), with and without contamination, and for discriminant rules based on the Biweight S-estimator, the Most Robust S-estimator (with breakdown point equal to 0.5), and the classical estimator. Note that we did not reporte the results for Case A, since they coincide with Case B-uncontaminated.

It can be clearly seen from Table 2 that there is a need to robustify discriminant analysis, since the misclassification probabilities under contamination of the classical procedures are always the biggest. If there is no contamination, then the 3 procedures give quite similar results, the classical estimator being marginally better. The results for the Biweight estimator and the restricted most B-robust estimator are comparable for the contaminated cases C and D, but the Biweight estimator gives significantly better results in the 2 other cases.

It is of course possible to construct worst case scenarios where the restricted most B-robust estimator would be better (otherwise it would not have the minimal gross-error sensitivity), but for many sampling configurations its optimality does not appear. One should not forget that most B-robust estimators gives only maximal protection against point contamination for small amount of contamination, and they are not guaranteed to be "most robust" in all situations.

Instead of taking the maximum of the influence function as a criterion to minimize, one could also think about other measures of robustness derived from the influence function. For example, as a referee suggested, the total area under the influence function could be considered.

Our results are limited to the linear discriminant rule of Fisher. Generalizations to a quadratic discriminant rule could be envisaged but they yield quite complicated expressions. Linear discriminant rules for the $k$-populations case, with $k>2$, have 
Table 2: Mean and Standard Deviation (STD) of the estimates of the Misclassification Error at four different sampling schemes of mixtures of normal distributions without contamination (u) and with contamination (c) for the Biweight S-estimator, the restricted most B-robust $S$-estimator and the classical estimator.

\begin{tabular}{|r|cc|cc|cc|}
\hline & \multicolumn{2}{|c|}{ Biweight S } & \multicolumn{2}{c|}{ Most Robust S } & \multicolumn{2}{c|}{ Classical Estimator } \\
& Mean & STD & Mean & STD & Mean & STD \\
\hline Case B u & 0.2046 & 0.000 & 0.2191 & 0.001 & 0.2004 & 0.000 \\
c & 0.2096 & 0.001 & 0.2915 & 0.004 & 0.6379 & 0.002 \\
Case C u & 0.2175 & 0.001 & 0.2302 & 0.002 & 0.2072 & 0.000 \\
c & 0.2612 & 0.004 & 0.2959 & 0.004 & 0.5989 & 0.001 \\
Case D u & 0.2253 & 0.001 & 0.2267 & 0.001 & 0.2106 & 0.001 \\
c & 0.2225 & 0.001 & 0.2312 & 0.002 & 0.2594 & 0.002 \\
Case E u & 0.3372 & 0.002 & 0.3372 & 0.002 & 0.3266 & 0.001 \\
c & 0.3872 & 0.002 & 0.4243 & 0.002 & 0.4294 & 0.001 \\
\hline
\end{tabular}

been robustified using M-estimators by Campbell (1982) and influence functions for the parameters of the discriminant function based on the classical estimators have been computed by Romanazzi (1991).

The results of Sections 2, 3, and 4 are valid for any affine equivariant location/scatter estimator, but the restricted and unrestricted Most Robust estimators were only obtained in the class of multivariate S-estimators. Of course, other classes of estimators of location/scatter are existing. While M-estimators are less attractive since they have a low breakdown point in high dimensions, this is no longer true for one-step versions of M-estimators, starting from a high-breakdown initial estimator. The asymptotics of these estimators have been considered in Lopuhaä (2000). In the context of linear regression this approach is very fruitful, since it combines high efficiency with high breakdown point (see Simpson, Ruppert \& Carroll 1992). For multivariate location and scatter problems, when $p>1$, S-estimators also succeed in combining good robustness and efficiency properties (see Tables 5 and 6 in Croux \& Haesbroeck 1999), so there seems to be less reason here to consider one-step M-estimators.

Another well-known location/scatter estimator is the Minimum Volume Ellipsoid (MVE) estimator (see Rousseeuw \& van Zomeren 1990). Strictly speaking, this estimator is an S-estimator with a $\rho$-function of the form $\rho(u)=I(|u|>c)$, for a certain constant $c$. Since this $\rho$-function is discontinuous, is does not verify the condition $(\mathbf{R})$. The MVE estimator is highly robust, but it has the drawback of having a slower convergence rate than the asymptotically normal S-estimators (Davies 1992). The computation of the MVE-estimator is much more demanding than that of an S-estimator. For the latter, the smoothness of the objective function can be used to construct fast algorithms, as in Ruppert (1992). The MVE-estimator has the maximal breakdown point of $50 \%$, but also a Biweight S-estimator and a restricted Most Robust S-estimator with this breakdown point are existing. Therefore we do not advice using the MVE-estimator for robustifying Fischer's linear discriminant rule.

An alternative for the MVE-estimator is the Minimum Covariance Determinant (MCD) estimator. This estimator has a $50 \%$ breakdown point, is asymptotically normal, and a fast algorithm exists to compute it (Rousseeuw \& Van Driessen 1999). The influence function for the location MCD-estimator is the same as the 
IF of an S-estimator with $\psi(t)=t I\left(|t| \leq \chi_{p, 0.5}\right)$, where $\chi_{p, 0.5}$ is the square root of the median of a chi-square distribution with $p$ degrees of freedom (see Butler, Davies \& Jhun 1993 ; Croux \& Haesbroeck 1999, Remark 4). Therefore the optimality result of Section 4 also applies for an MCD based procedure.

Finally we should mention a completely different approach to robust discriminant analysis, based on projection pursuit. The idea here is to project the data on the direction $\lambda$ such that the the 2 groups of projected data are the most distinguishable. When using the two-sample t-test as a measure of difference between the 2 projected groups, we find Fischer's rule. Using other measures yield other discriminant analysis rules (Posse 1992; Van Ness \& Yang 1998). Although intuitively appealing, this projection pursuit approach to robust discriminant analysis will be highly time consuming and not much theory seems to have been developed in this area.

\section{APPENDIX}

Proof of Lemma 1: Let $\varepsilon>0$, and $H_{\varepsilon}=(1-\varepsilon) H_{0}+\varepsilon \Delta_{x_{0}}$. Suppose wlog $x_{0} \in \wp_{1}$. Then

$$
\begin{aligned}
H_{\varepsilon} & =(1-\varepsilon) p_{1} H_{1}+(1-\varepsilon) p_{2} H_{2}+\varepsilon \Delta_{x_{0}} \\
& =\left\{(1-\varepsilon) p_{1}+\varepsilon\right\}\left[\left\{1-\frac{\varepsilon}{(1-\varepsilon) p_{1}+\varepsilon}\right\} H_{1}+\frac{\varepsilon}{(1-\varepsilon) p_{1}+\varepsilon} \Delta_{x_{0}}\right]+(1-\varepsilon) p_{2} H_{2} \\
& =p_{1}(\varepsilon)\left\{(1-\delta(\varepsilon)) H_{1}+\delta(\varepsilon) \Delta_{x_{0}}\right\}+p_{2}(\varepsilon) H_{2}
\end{aligned}
$$

with $p_{1}(\varepsilon)=(1-\varepsilon) p_{1}+\varepsilon, \delta(\varepsilon)=\frac{\varepsilon}{p_{1}(\varepsilon)}$ and $p_{2}(\varepsilon)=(1-\varepsilon) p_{2}$. From definition (4) it follows

$$
C_{P}\left(H_{\varepsilon}\right)=p_{1}(\varepsilon) C\left\{(1-\delta(\varepsilon)) H_{1}+\delta(\varepsilon) \Delta_{x_{0}}\right\}+p_{2}(\varepsilon) C\left(H_{2}\right)
$$

Differentiating (18) with respect to $\varepsilon$ and evaluating at zero yields

$$
\begin{aligned}
I F\left(x_{0}, C_{P}, H_{0}\right) & =\left(1-p_{1}\right) C\left(H_{1}\right)+\left.p_{1} I F\left(x_{0}, C, H_{1}\right) \frac{\partial}{\partial \varepsilon} \delta(\varepsilon)\right|_{\varepsilon=0}-p_{2} C\left(H_{2}\right) \\
& =\left(1-p_{1}\right) \Sigma+p_{1} I F\left(x_{0}, C, H_{1}\right) \frac{1}{p_{1}}-p_{2} \Sigma \\
& =\operatorname{IF}\left(x_{0}, C, H_{1}\right) .
\end{aligned}
$$

Proof of Proposition 1: From the definition of the functional $l(G)$, Lemma 1, equations (7) and (8), and the fact that $\operatorname{IF}\left(x_{0}, C^{-1}, H_{0}\right)=-\Sigma^{-1} I F\left(x_{0}, C, H_{0}\right) \Sigma^{-1}$, we obtain

$$
\begin{aligned}
I F\left(x_{0}, l, H_{0}\right)= & -\Sigma^{-1} I F\left(x_{0}, C, H\right) \Sigma^{-1}\left(\mu_{1}-\mu_{2}\right)+(-1)^{j+1} \Sigma^{-1} I F\left(x_{0}, T, H_{j}\right) \\
= & \left\{\alpha\left(d_{\mu_{j}, \Sigma}^{2}\left(x_{0}\right)\right) \Sigma^{-1}\left(x_{0}-\mu_{j}\right)\left(x_{0}-\mu_{j}\right)^{t}-\beta\left(d_{\mu_{j}, \Sigma}^{2}\left(x_{0}\right)\right)\right\} \Sigma^{-1}\left(\mu_{2}-\mu_{1}\right) \\
& +(-1)^{j+1} \zeta\left(d_{\mu_{j}, \Sigma}^{2}\left(x_{0}\right)\right) \Sigma^{-1}\left(x_{0}-\mu_{j}\right) .
\end{aligned}
$$

By using the above equation and (2), the equality stated in the Proposition follows.

Proof of Proposition 2: By equation (5) and Proposition 1, it follows 


$$
\begin{aligned}
I F( & \left.x_{0}, L_{x}, H_{0}\right)=\left(x-\frac{\mu_{1}+\mu_{2}}{2}\right)^{t} I F\left(x_{0}, l, H_{0}\right)-\frac{1}{2} \lambda^{t} I F\left(x_{0}, T, H_{j}\right) \\
= & \left(x-\frac{\mu_{1}+\mu_{2}}{2}\right)^{t}\left\{\beta\left(d_{\mu_{j}, \Sigma}^{2}\left(x_{0}\right)\right) \lambda-\alpha\left(d_{\mu_{j}, \Sigma}^{2}\left(x_{0}\right)\right) \Sigma^{-1}\left(x_{0}-\mu_{j}\right)\left(x_{0}-\mu_{j}\right)^{t} \lambda\right. \\
& \left.+(-1)^{j+1} \zeta\left(d_{\mu_{j}, \Sigma}^{2}\left(x_{0}\right)\right) \Sigma^{-1}\left(x_{0}-\mu_{j}\right)\right\}-\frac{1}{2} \lambda^{t} \zeta\left(d_{\mu_{j}, \Sigma}^{2}\left(x_{0}\right)\right)\left(x_{0}-\mu_{j}\right) \\
= & \beta\left(d_{\mu_{j}, \Sigma}^{2}\left(x_{0}\right)\right) \Lambda(x)-\alpha\left(d_{\mu_{j}, \Sigma}^{2}\left(x_{0}\right)\right)\left(x-\frac{\mu_{1}+\mu_{2}}{2}\right)^{t} \Sigma^{-1}\left(x_{0}-\mu_{j}\right)\left(x_{0}-\mu_{j}\right)^{t} \lambda \\
& -\zeta\left(d_{\mu_{j}, \Sigma}^{2}\left(x_{0}\right)\right)\left\{\frac{\lambda^{t}}{2}\left(x_{0}-\mu_{j}\right)+(-1)^{j}\left(x-\frac{\mu_{1}+\mu_{2}}{2}\right)^{t} \Sigma^{-1}\left(x_{0}-\mu_{j}\right)\right\} .
\end{aligned}
$$

Proof of Proposition 3: Since $\Pi_{12}\left(\varepsilon, x_{0}, H\right)=P\left(l\left(H_{\varepsilon}\right)^{t} X-k\left(H_{\varepsilon}\right)<0 \mid X \sim\right.$ $\left.N\left(\mu_{1}, \Sigma\right)\right)$ with $H_{\varepsilon}=(1-\varepsilon) H_{0}+\varepsilon \Delta_{x_{0}}$, it follows that

$$
\begin{aligned}
\Pi_{12}\left(\varepsilon, x_{0}, H\right) & =P\left(l\left(H_{\varepsilon}\right)^{t}\left(\Sigma^{1 / 2} U+\mu_{1}\right)-k\left(H_{\varepsilon}\right)<0 \mid U \sim N\left(0, I_{p}\right)\right) \\
& =P\left(\sqrt{l\left(H_{\varepsilon}\right)^{t} \Sigma l\left(H_{\varepsilon}\right)} Z+l\left(H_{\varepsilon}\right)^{t} \mu_{1}-k\left(H_{\varepsilon}\right)<0 \mid Z \sim N(0,1)\right) \\
& =\Phi\left(\frac{k\left(H_{\varepsilon}\right)-\mu_{1}^{t} l\left(H_{\varepsilon}\right)}{\sqrt{l\left(H_{\varepsilon}\right)^{t} \Sigma l\left(H_{\varepsilon}\right)}}\right),
\end{aligned}
$$

where $\Phi$ is the cumulative standard normal distribution function. Deriving the last expression with respect to $\varepsilon$ and evaluating at zero yields

$$
\begin{aligned}
I F\left(x_{0}, \Pi_{12}, H\right)= & \phi\left(\frac{k-\mu_{1}^{t} \lambda}{\sqrt{\lambda^{t} \Sigma \lambda}}\right) \frac{1}{\lambda^{t} \Sigma \lambda}\left\{\left.\frac{\partial}{\partial \varepsilon}\left(k\left(H_{\varepsilon}\right)-\mu_{1}^{t} l\left(H_{\varepsilon}\right)\right)\right|_{\varepsilon=0} \sqrt{\lambda^{t} \Sigma \lambda}\right. \\
& \left.+\left.\left(\mu_{1}^{t} l-k\right) \frac{\partial}{\partial \varepsilon} \sqrt{l\left(H_{\varepsilon}\right)^{t} \Sigma l\left(H_{\varepsilon}\right)}\right|_{\varepsilon=0}\right\} \\
= & \frac{\phi\left(\frac{k-\mu_{1}^{t} \lambda}{\sqrt{\lambda^{t} \Sigma \lambda}}\right)}{\sqrt{\lambda^{t} \Sigma \lambda}}\left\{-I F\left(x_{0}, L_{\mu_{1}}, H_{0}\right)+\Lambda\left(\mu_{1}\right) \frac{\lambda^{t} \Sigma I F\left(x_{0}, l, H_{0}\right)}{\lambda^{t} \Sigma \lambda}\right\} .
\end{aligned}
$$

But by (6) we have $I F\left(x_{0}, L_{\mu_{1}}, H_{0}\right)=\left(\frac{\mu_{1}-\mu_{2}}{2}\right)^{t} I F\left(x_{0}, l, H\right)-\frac{\lambda^{t}}{2} I F\left(x_{0}, T, H_{j}\right)$. Moreover $\Lambda\left(\mu_{1}\right)=\frac{1}{2} \lambda^{t} \Sigma \lambda$, so

$$
\begin{aligned}
I F\left(x_{0}, \Pi_{12}, H\right) & =\frac{\phi\left(\frac{k-\mu_{1}^{t} \lambda}{\sqrt{\lambda^{t} \Sigma \lambda}}\right)}{2 \sqrt{\lambda^{t} \Sigma \lambda}}\left[\left\{\lambda^{t} \Sigma-\left(\mu_{1}-\mu_{2}\right)^{t}\right\} I F\left(x_{0}, l, H_{0}\right)+\lambda^{t} I F\left(x_{0}, T, H_{j}\right)\right] \\
& =\frac{\phi\left(\frac{k-\mu_{1}^{t} \lambda}{\sqrt{\lambda^{t} \Sigma \lambda}}\right)}{\sqrt{\lambda^{t} \Sigma \lambda}} \frac{\zeta\left(d_{\mu_{j}, \Sigma}^{2}\right)}{2} \lambda^{t}\left(x_{0}-\mu_{j}\right)
\end{aligned}
$$

because $\operatorname{IF}\left(x_{0}, T, H_{j}\right)=\zeta\left(d_{\mu_{j}, \Sigma}^{2}\right)\left(x_{0}-\mu_{j}\right)$ and $\Sigma \lambda=\mu_{1}-\mu_{2}$. The result follows now directly by noting that $\lambda^{t} \Sigma \lambda=d_{\mu_{1}, \Sigma}^{2}\left(\mu_{2}\right)=d_{\mu_{2}, \Sigma}^{2}\left(\mu_{1}\right)$.

Proof of Lemma 2: By partial integration we have

$$
\int_{0}^{\infty} G_{\alpha}(r) \psi(r) d r=\rho(\infty) G_{\alpha}(\infty)-\int_{0}^{\infty} \rho(r) d G(r)=\alpha \rho(\infty)-E_{G}[\rho(Y)] .
$$

The results follow now from the definition of $G$.

Proof of Theorem 1: As outlined in Section 4, it is sufficient to prove that $\psi^{*}$ solves problem $(\mathbf{P})$. Take $\psi$ an arbritary function satisfying $(\mathbf{\Psi})$ with corresponding breakdown point $\varepsilon \geq \varepsilon^{*}$. The $\psi$-function is standardized such that

$$
\sup _{t \geq 0} \psi(t)=1
$$


From (15) and Lemma 2 we know that for an $\alpha \geq \varepsilon *$, we have

$$
\int_{0}^{\infty} G_{\alpha}(r) \psi(r) d r=0
$$

In fact, this $\alpha$ equals $\varepsilon$ or $1-\varepsilon$. Using (19), it is clear that it suffices to prove that $\int_{0}^{\infty} \psi(r) \xi(r) d r \leq \int_{0}^{c^{*}} \xi(r) d r$ with $c^{*}=c_{\varepsilon^{*}}$, and $\xi(r)=-g^{\prime}\left(r^{2}\right) r^{p}$. We can see that $\xi(r)$ has only one maximum $r^{*}$, equal to $\sqrt{p}$. Before $r^{*}$ the function $\xi(r)$ is strictly increasing, afterwards strictly decreasing.

Let $c=\inf \{s \mid \psi(t)=0 \forall t \geq s\}$. If $c \leq c^{*}$, then it obviously holds that

$$
\int_{0}^{\infty} \psi(r) \xi(r) d r=\int_{0}^{c} \psi(r) \xi(r) d r \leq \int_{0}^{c} \xi(r) d r \leq \int_{0}^{c^{*}} \xi(r) d r .
$$

Suppose now that $c>c^{*}$. We will show that in this case $\psi$ can never be a solution of problem $(\mathbf{P})$, which will also end the proof. Suppose for a moment that $\psi$ is a solution of $(\mathbf{P})$. Let $t_{\alpha}=G^{-1}(1-\alpha)$. Of course $G_{\alpha}\left(t_{\alpha}\right)<0$ for $t<t_{\alpha}$ and $G_{\alpha}\left(t_{\alpha}\right)>0$ for $t>t_{\alpha}$. Also $c_{\alpha}>t_{\alpha}$.

First of all, we may suppose that $\psi(r)=1$ for any $0 \leq r \leq t_{\alpha}$. If not, we can construct a function $\psi_{1}$ as

$$
\psi_{1}(r)=\left\{\begin{array}{ll}
1 & \text { for } r \in\left[0, t_{\alpha}\right] \\
\psi(r) & \text { for } \left.r \in] t_{\alpha}, c\right] \\
1 & \text { for } \left.r \in] c, c_{1}\right] \\
0 & \text { for } r>c_{1}
\end{array},\right.
$$

where $c_{1}$ is defined as the solution of

$$
\int_{c}^{c_{1}} G_{\alpha}(r) d r=\int_{0}^{t_{\alpha}}(\psi(r)-1) G_{\alpha}(r) d r
$$

since $G_{\alpha}(r)$ is negative on $\left[0, t_{\alpha}[\right.$ and $\psi(r) \leq 1$ with strict inequality on a non negligeable set, we have $c_{1}>c$. It is easy to verify that $\int_{0}^{\infty} \psi_{1}(r) G_{\alpha}(r) d r=0$ while at the same time

$$
\int_{0}^{\infty} \psi(r) \xi(r) d r<\int_{0}^{\infty} \psi_{1}(r) \xi(r) d r
$$

contradicting that $\psi$ solves problem $(\mathbf{P})$.

Define now for each $\delta>0$ the sets $\left.\left.\tilde{D}_{\delta}=\{r \in] c^{*}, c\right] ; \psi(r)>\delta\right\}$ and $\tilde{E}_{\delta}=\{r \in$ ]$\left.\left.t^{*}, c^{*}\right] ; \psi(r)<1-\delta\right\}$, with $t^{*}=t_{\varepsilon^{*}}=G^{-1}\left(1-\varepsilon^{*}\right)$. There exist a $\delta_{1}>0$ such that $\tilde{E}_{\delta_{1}}$ is not empty. Otherwise we would have $\psi(r)=1$ on $\left[t^{*}, c^{*}\right]$. But then, since $t_{\alpha} \leq t^{*}, c^{*}<c$ and $G_{\varepsilon^{*}}(r) \leq G_{\alpha}(r)$ for every $r \geq 0$, the inequalities

$$
\begin{aligned}
\int_{t_{\alpha}}^{c} G_{\alpha}(r) \psi(r) d r & >\int_{t_{\alpha}}^{c^{*}} G_{\alpha}(r) \psi(r) d r \geq \int_{t^{*}}^{c^{*}} G_{\alpha}(r) d r \\
& \geq \int_{t^{*}}^{c^{*}} G_{\varepsilon^{*}}(r) d r=-\int_{0}^{t^{*}} G_{\mathcal{\varepsilon}^{*}}(r) d r
\end{aligned}
$$

would hold. We also have,

$$
\int_{0}^{t^{*}} G_{\varepsilon^{*}}(r) d r \leq \int_{0}^{t_{\alpha}} G_{\alpha}(r) d r=\int_{0}^{t_{\alpha}} G_{\alpha}(r) \psi(r) d r .
$$


Combining (20) and (21) would therefore to a contradiction since $\psi$, due to (19), needs to verify

$$
\int_{t_{\alpha}}^{c} G_{\alpha}(t) \psi(t) d t=-\int_{0}^{t_{\alpha}} G_{\alpha}(t) \psi(t) d t
$$

so that we may conclude that there exist a $\delta_{1}>0$ such that $\tilde{E}_{\delta_{1}}$ is not empty. By definition of $c$, we may assume that there exists a further $0<\delta_{2} \leq \delta_{1}$ such that both $\tilde{E}_{\delta_{2}}$ and $\tilde{D}_{\delta_{2}}$ are non empty.

Select then two subintervals $D_{\delta}$ and $E_{\delta}$ from $\tilde{D}_{\delta_{2}}$ and $\tilde{E}_{\delta_{2}}$ of the same length. Define

$$
\psi_{2}(t)= \begin{cases}\psi(t)-\delta & \text { for } t \in D_{\delta} \\ \psi(t)+\delta & \text { for } t \in E_{\delta} \\ \psi(t) & \text { otherwise. }\end{cases}
$$

By construction, $\psi_{2}(t) \leq 1$ everywhere. Moreover, by tedious calculation it can be checked that it holds (for the normal distribution) that for every $r>c_{\alpha}: \xi(r)<$ $\xi\left(t_{\alpha}\right)$, implying

$$
\sup _{r \in D_{\delta}} \xi(r)<\xi\left(c^{*}\right) \leq \inf _{r \in E_{\delta}} \xi(r)
$$

But then

$$
\int_{D_{\delta}} \xi(r) d r<\int_{E_{\delta}} \xi(r) d r
$$

yielding

$$
\int_{0}^{c} \psi_{2}(r) \xi(r) d r>\int_{0}^{c} \psi(r) \xi(r) d r
$$

Also,

$$
\begin{aligned}
\int_{t^{*}}^{c} G_{\alpha}(r) \psi_{2}(r) d r & =\int_{t^{*}}^{c} G_{\alpha}(r) \psi(r) d r+\delta\left[\int_{E_{\delta}} G_{\alpha}(r) d r-\int_{D_{\delta}} G_{\alpha}(r) d r\right] \\
& <\int_{t^{*}}^{c} G_{\alpha}(r) \psi(r) d r
\end{aligned}
$$

since $G_{\alpha}(r)$ is increasing, $\sup E_{\delta} \leq \inf D_{\delta}$ and the fact that the intervals $E_{\delta}$ and $D_{\delta}$ have the same the same length. But then $\int_{0}^{c} G_{\alpha}(r) \psi_{2}(r) d r \leq 0$ and it will be possible to construct a $\psi_{3}(r)$ with $\int_{0}^{\infty} G_{\alpha}(r) \psi_{3}(r) d r=0, \psi_{3}(r)=\psi_{2}(r)$ on $[0, c], \sup _{r} \psi_{3}(r)=1$, and $\int_{0}^{\infty} \xi(r) \psi_{3}(r) d r \geq \int_{0}^{\infty} \xi(r) \psi_{2}(r) d r>\int_{0}^{\infty} \psi(r) \xi(r) d r$. Therefore, $\psi$ does not solve $(\mathbf{P})$ and the case $c>c^{*}$ can be excluded.

\section{ACKNOWLEDGEMENTS}

We would like to thank the associate editor and the referees for helpful comments which improved significantly the presentation of this paper.

\section{REFERENCES}

R. W. Butler, P. L. Davies \& M. Jhun (1993). Asymptotics for the Minimum Covariance Determinant Estimator. The Annals of Statistics, 21, 1385-1400.

N. A. Campbell (1978). The influence function as an aid in outlier detection in discriminant analysis. Applied Statistics, 27, 251-258.

N. A. Campbell (1982). Robust Procedures in Multivariate Analysis: Robust Canonical Variate Analysis. Applied Statistics, 31, 1-8. 
C. Y. Chork \& P. J. Rousseeuw (1992). Integrating a High-Breakdown option into Discriminant Analysis in Exploration Geochemistry. Journal of Geochemical Exploration, 43, 191-203.

F. Critchley \& C. Vitiello (1991). The Influence of Observations on Misclassification Probability Estimates in Linear Discriminant Analysis. Biometrika, 78, 677-690.

C. Croux \& G. Haesbroeck (1999). Influence Function and Efficiency of the Minimum Covariance Determinant Scatter Matrix Estimator. The Journal of Multivariate Analysis, 71,161-190.

C. Croux \& G. Haesbroeck (1999). Principal Component Analysis based on Robust Estimators of the Covariance or Correlation Matrix: Influence Functions and Efficiencies. Biometrika, 87, 603-618.

C. Croux, C. Dehon, P. J. Rousseeuw \& S. Van Aelst (2000). Robust Estimation of the Conditional Median Function at Elliptical Models. Statistics and Probability Letters, 51,361-368.

P. L. Davies (1987). Asymptotic Behavior of S-Estimators of Multivariate Location Parameters and Dispersion Matrices. The Annals of Statistics, 15, 1269-1292.

P. L. Davies (1992). The asymptotics of Rousseeuw's minimum volume ellipsoid estimator. The Annals of Statistics, 4, 1828-1843.

F. R. Hampel, E. M. Ronchetti, P. J. Rousseeuw \& W. A. Stahel (1986). Robust Statistics: The Approach Based in Influence Functions. New York: John Wiley and Sons.

D. M. Hawkins \& G. J. McLachen (1997). High-Breakdown Linear Discriminant Analysis. Journal of the American Statistical Association, 92, 136-143.

X. He \& W. K. Fung (2000). High Breakdown Estimation for Multiple Populations with Applications to Discriminant Analysis. Journal of Multivariate Analysis, 72, 151-162.

O. Hössjer (1992). On the Optimality of S-estimators. Statistics and Probability Letters, 14, 413-419.

R. A. Johnson \& D. W. Wichern (1998). Applied Multivariate Statistical Analysis, fourth edition. Prentice Hall International Editions.

P. A. Lachenbruch (1966). Discriminant Analysis When the Initial Samples Are Misclassified. Technometrics, 8, 657-662.

H. P. Lopuhaä (1989). On the Relation Between S-estimators and M-estimators of Multivariate Location and Covariance. The Annals of Statistics, 17, 1662-1683.

H. P. Lopuhaä (2000). Asymptotics of Reweighted Estimators of Multivariate Location and Scatter. The Annals of Statistics, 27, 1638-1665.

R. A. Maronna (1976). Robust M-estimators of Multivariate Location and Scatter. The Annals of Statistics, 4, 51-67.

C. Posse (1992). Projection Pursuit Discriminant Analysis for two Groups. Communication Statistics - Theory Methods, 21, 1-19.

R. H. Randles, J. D. Broffitt, J. R. Ramberg \& R. V. Hogg (1978). Generalized Linear and Quadratic Discriminant Functions Using Robust Estimates. Journal of the American Statistical Association, 73, 564-568.

M. Romanazzi (1991). Influence in Canonical Variates Analysis. Computational Statistics and Data Analysis, 11, 143-164.

P. J. Rousseeuw (1982). Most robust M-estimators in the infinitesimal sense. Zeitschrift für Wahrscheinlichkeitstheorie und Verwandte Gebiete, 61, 541-551.

P. J. Rousseeuw \& A. M. Leroy (1987). Robust Regression and Outlier Detection, New York: John Wiley. 
P. J. Rousseeuw \& B. C. van Zomeren (1990). Unmasking multivariate outliers and leverage points. Journal of the American Statistical Association, 85, 663-639.

P. J. Rousseeuw \& K. Van Driessen (1999). A fast algorithm for the minimum covariance determinant estimator. Technometrics 41, 212-23.

D. Ruppert (1992). Computing S-estimators for regression and multivariate location and dispersion. Journal of Computational and Graphical Statistics, 1, 253-270.

D. G. Simpson, D. Ruppert \& R. J. Carroll (1992). On one-step GM estimates and stability of inferences in linear regression. Journal of the American Statistical Association, 87, 439-450.

J. W. Van Ness \& J. J. Yang (1998). Robust discriminant analysis: Training data breakdown point. Journal of Statistical Planning and Inference, 67, 67-83.

Received ???

Accepted ???
Christophe CROUX: ccroux@ll b. ac. be ECARES, Faculté SOCO and Institut de Statistique Université Libre de Bruxelles B-1050 Bruxelles Belgium.

Catherine DEHON: cdehon@il b. ac. be ECARES, Faculté SOCO and Institut de Statistique Université Libre de Bruxelles B-1050 Bruxelles Belgium. 